C-A/AP/\#158 July 2004

\title{
Skew Quadrupole Modulation to Measure Global Betatron Coupling
}

\author{
Y. Luo, P. Cameron, R. Lee, A. Marusic, F. Pilat, \\ D. Trbojevic, T. Roser, J. Wei
}

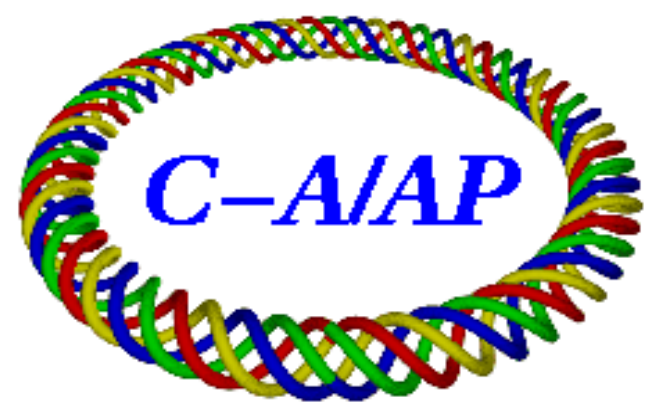

Collider-Accelerator Department Brookhaven National Laboratory Upton, NY 11973 


\title{
Skew Quadrupole Modulation to Measure Global Betatron Coupling*
}

\author{
Y. Luo, P. Cameron, R. Lee, A. Marusic, F. Pilat, D. Trbojevic, T. Roser, J. Wei \\ Brookhaven National Laboratory, Upton, NY 11973, USA
}

\begin{abstract}
The measurement of the residual betatron coupling via skew quadrupole modulation is a new diagnostics technique that has been developed and tested at the Relativistic Heavy Ion Collider (RHIC) as a promising method for the linear decoupling on the ramp. By modulating the strengths of different skew quadrupole families the two eigentunes are precisely measured with the phase lock loop system. The projections of the residual coupling coefficient onto the skew quadrupole coupling modulation directions are determined. The residual linear coupling could be corrected according to the measurement. An analytical solution for skew quadrupole modulation based on the Hamiltonian perturbation theory is given, and simulation code using smooth accelerator model is also developed. Some issues concerning the practical applications of this technique are discussed. In the second half of this note, We report the results from the dedicated beam studies carried on at the RHIC injection, store and on the ramp. A capability of measuring coupling on the ramp opens possibility of continuous coupling corrections during acceleration.
\end{abstract}

\section{INTRODUCTION}

For a hadron machine like RHIC, the working points are constrained in a very narrow space. It is demanded to keep the two tunes close to the linear difference resonance line in order to get better beam lifetime. RHIC presently has been equipped with applications for static linear coupling correction at injection and store. However, the coupling correction on the ramp is still an open problem, which become more and more important in the past few years, especially during the polarized proton operations. In order to avoid polarization loss, it is essential to keep the tunes almost constant on the ramp for RHIC polarized proton operation. The coupling changes on the ramp with variations of beam optics and closed orbit distortion. A fast, robust mechanism and a set of reliable diagnostics are needed to accomplish the task.

A skew quadrupole strength scan is the general way to decouple the machine globally. However, it is not suitable for coupling correction on the ramp because of the needs of moving tunes to get the minimum separation before correction, slow and large range scan to find the correction strength, bad resolution to determine the minimum tune separation, and possibility of causing beam abortion during scan. As a logical extension, T. Roser suggested skew quadrupole modulation to replace the skew quadrupole

\footnotetext{
* Work supported by U.S. DOE under contract No DE-AC0298CH10886
}

scan for the ramp global coupling correction [1]. The basic point is to reduce the amplitude ratio of $1 f$ peak to $2 f$ peak in the fast fourier transform (FFT) plot of $\left(Q_{1}-Q_{2}\right)^{2}$, where the $f$ is the skew quadrupole strength modulation frequency, $Q_{1}, Q_{2}$ are two tunes' decimal parts. The idea is proved in the analytical solution shown below. To track the tune changes during modulation, phase lock loop ( PLL ) tune measurement data are used. The PLL system has been commissioned in RHIC for years and has become an indispensable tool in the routine performance of RHIC. This article focuses on the analytical solution, simulation study and discussions about the use of this technique. Results from beam experiments in RHIC are given in the second half of this note.

\section{ANALYTICAL SOLUTION}

There are several approaches to tackle the linear coupling. Since we are interested in the two global tunes' response to the skew quadrupole modulation, perturbation theory $[2,3]$ based on Hamiltonian mechanism is more straight and simple and is therefore adopted here. The two eigentunes $Q_{1}, Q_{2}$ are given by

$$
\begin{aligned}
& Q_{1}=Q_{x, 0}-\frac{\Delta}{2}+\frac{1}{2} \sqrt{\Delta^{2}+\left(C^{-}\right)^{2}}, \\
& Q_{2}=Q_{y, 0}+\frac{\Delta}{2}-\frac{1}{2} \sqrt{\Delta^{2}+\left(C^{-}\right)^{2}} .
\end{aligned}
$$

the tune separation's square is

$$
\left(Q_{1}-Q_{2}\right)^{2}=\Delta^{2}+\left(C^{-}\right)^{2},
$$

where $Q_{x, 0}$ and $Q_{y, 0}$ are the uncoupled tunes without any couplers, $\Delta$ is the distance to the linear difference coupling resonance, $C^{-}$is the total coupling coefficient at one observation point, which normally is a complex number. $\Delta$ and $C^{-}$are defined as:

$$
\begin{aligned}
\Delta & =Q_{x, 0}-Q_{y, 0}-p, \\
C^{-} & =\frac{1}{2 \pi} \oint \sqrt{\beta_{x} \beta_{y}} k_{s} e^{i\left(\Psi_{x}-\Psi_{y}-\Delta \frac{2 \pi s}{C}\right)} d l .
\end{aligned}
$$

In order to distinguish the different sources of coupling coefficient, in the following we design $C_{t o t}^{-}$as the total coupling coefficient, $C_{\text {res }}^{-}$the residual coupling coefficient, $C_{m o d}^{-}$the induced coupling coefficient by the skew quadrupole modulation. To simplify narration, we will not distinguish the coupling coefficient and coupling, either.

If one skew quadrupole's current modulates, so does the induced coupling too. Assuming the modulation amplitude of the coupling is $C_{m o d, a m p}^{-}$, the induced and total coupling are given by

$$
C_{m o d}^{-}=C_{m o d, a m p}^{-} \sin (2 \pi f t)
$$




$$
C_{\text {tot }}^{-}=C_{\text {res }}^{-}+C_{\text {mod }}^{-}
$$

We introduce the slow varying approximation, that is, the skew quadrupole modulation frequency is slow enough so that the eigentunes are only determined by the modulation strength. Of course, the modulation amplitude should be small so that the above perturbation theory still holds. Substituting Eq. 6 into Eq. 3, we get

$$
\begin{aligned}
\left(Q_{1}-Q_{2}\right)^{2}= & \Delta^{2}+\left|C_{\text {res }}^{2}\right|^{2}+\frac{1}{2}\left|C_{\text {mod,amp }}^{2}\right|^{2} \\
& +2\left|C_{\text {res }}^{-}\right|\left|C_{\text {mod,amp }}^{-}\right| \cos (\varphi) \sin (2 \pi f t) \\
& -\frac{1}{2}\left|C_{\text {mod,amp }}^{-}\right|^{2} \cos (4 \pi f t)
\end{aligned}
$$

where the $\varphi$ is the angle difference between $C_{\text {res }}^{-}$and $C_{\text {mod,amp. }}\left|C_{\text {res }}^{-}\right| \cos (\varphi)$ is the projection of the residual coupling onto the modulation coupling.

Now it is clear from Eq. 8 that the $2 f$ item is only related to skew quadruple modulation amplitude, the $1 f$ item is related to the dot multiplication of the modulation coupling and the residual coupling. In the frequency domain of $\left(Q_{1}-Q_{2}\right)^{2}$ during skew quadruploe modulation, we will see two peaks located at $1 f$ and $2 f$ if the machine is originally coupled, or only the $2 f$ peak if the machine originally well decoupled. So the $1 f$ peak is one reflection, or observable of the global residual coupling in one accelerator.

In a real machine it is therefore useful to use this technique to do fast measurements of residual coupling with two or more different skew quadruple modulations at injection, store and hopefully on the ramp. Knowing the residual coupling's amplitude and angle, it is possible to carry out global coupling correction with known skew quadrupole correctors. This correction technique could be used in feed forward or feedback scenarios.

\section{SIMULATION}

With the above analytical solution, a simulation program has been developed based on smooth accelerator model. The uncoupled motion is simply represented by

$$
\begin{aligned}
& x^{\prime \prime}+\left(\frac{Q_{x, 0}}{R}\right)^{2} x=0, \\
& y^{\prime \prime}+\left(\frac{Q_{y, 0}}{R}\right)^{2} y=0 .
\end{aligned}
$$

where $R$ is the average radius of the circular accelerator. The artificial skew quadrupoles with zero length are distributed along the ring, the $4 \times 4$ transfer matrix between skew quadrupoles are diagonalized.

For each RHIC ring, there are three families of correction skew quadrupoles due to the six-fold structure of the ring. If appropriately powered, the coupling coefficients from the three families are $120^{\circ}$ apart. So in the simulation, we insert three skew quadrupoles equidistantly in the ring model. Each quadrupole could have constant, modulated strengths, or both. The constant strengths of three skew quadrupoles present the residual coupling. The uncoupled tunes are $\left(Q_{x, 0}, Q_{y, 0}\right)=(28.22,29.23)$, the average radius $R=C / 2 \pi, C$ is the RHIC ring circumference $3833.84 \mathrm{~m}$. For every tracking, one particle with initial coordinates is launched at the location of first skew quadrupole and successive particle $x$ and $y$ coordinates are recorded at the third quadrupole location.

Fig. 1 shows the two tunes and Fig. 2 is the FFT of the $\left(Q_{1}-Q_{2}\right)^{2}$ from simulation when the third skew quadrupole strength modulates with integrated strength amplitude $\left(\left(k_{s} d l\right)_{3}\right)_{a m p, \text { mod }}=0.0005 \mathrm{~m}^{-1}$, modulation frequency $0.5 \mathrm{~Hz}$, under condition that the first and the second skew quadruple strengths are constant at $\left(k_{s} d l\right)_{1,2}=$ $0.0005 \mathrm{~m}^{-1}$.

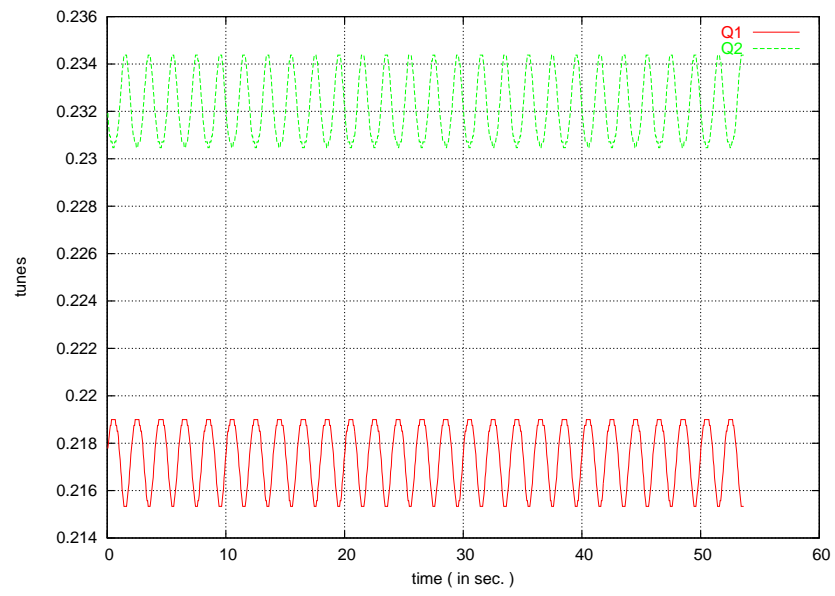

Figure 1: An example of tunes from simulation during skew quadruploe modulation. Every set of tunes $\left(Q_{1}, Q_{2}\right)$ are achieved from FFT of 4096 turns' $(x+y)$ data.

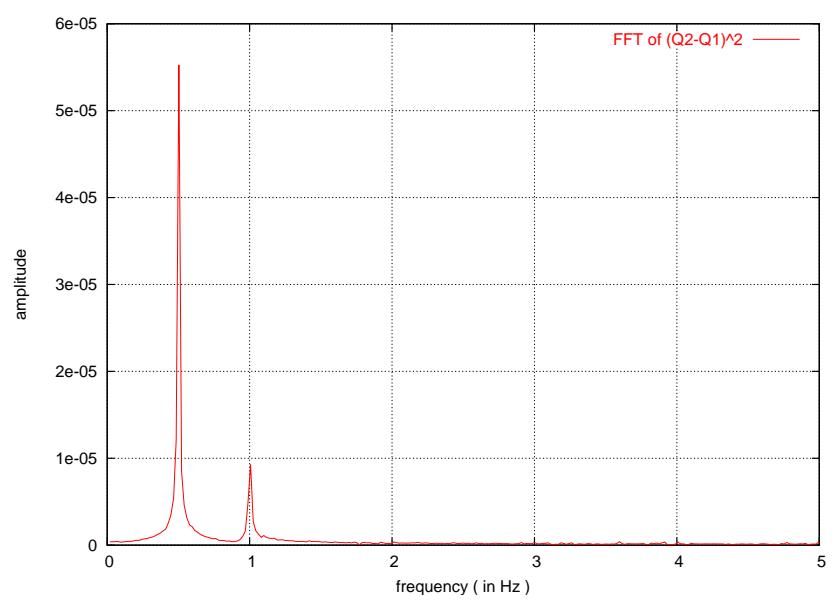

Figure 2: An example of $\left(Q_{2}-Q_{1}\right)^{2}$ FFT spectrum from simulation. This plot is obtained from 1024 sets of tunes from Fig. 1.

\section{DISCUSSION}

In the following we discuss how to use the results from the skew quadrupole modulation and some specific issues 
connecting its practical applications.

\section{Projection Ratio}

A straight use of Eq. 8 is the projection ratio of the residual coupling coefficient onto the modulation coupling direction. It is defined as:

$$
\kappa=\frac{\left|C_{\text {res }}^{-}\right| \cos (\varphi)}{\left|C_{\text {mod,amp }}^{-}\right|} .
$$

$\kappa$ is a dimensionless quantity, however it has its sign which is decided by the difference angle $\varphi$ between the residual and modulation couplings. From FFT of $\left(Q_{1}-Q_{2}\right)^{2}$, we could get $1 f$ and $2 f$ peaks' amplitudes, say $A_{1 f}, A_{2 f}$, then

$$
|\kappa|=\left(\frac{A_{1 f}}{A_{2 f}}\right) / 4 .
$$

$\kappa$ 's sign is same to that of $\sum_{i=1}^{N}\left(Q_{1}-Q_{2}\right)_{i}^{2} \times i_{\text {mod,courrent }}$ , where $I_{0} \sin (2 \pi f t)$ is skew quadrupole modulation power supply current, $I_{0}$ is normally positive.

\section{Modulation Strength and Frequency}

The skew quadrupole modulation have the advantage over skew quadrupole scans that the modulation strength could be small in operation. It is very important not to deteriorate the beam lifetime during measurement. Eq. 11 shows that the $1 f$ peak amplitude is enlarged by 4 times comparing to the $2 f$ peak in the $\left(Q_{1}-Q_{2}\right)^{2}$ FFT plot if the residual and induced couplings' amplitudes are the same. Skew quadrupole modulation is eligible to measure small residual coupling.

The skew quadrupole modulation frequency is chosen between $0.2-1.0 \mathrm{~Hz}$ for RHIC to fulfil the slow varying approximation and system limitation in the power supply system and PLL response. It has be shown from simulation and beam experiment that there will be spurious peaks in the $\left(Q_{1}-Q_{2}\right)^{2}$ FFT plot, multipoles of modulation frequencies if the frequency is too large. On the other hand, if the modulation is too slow, the measurement can be too long to accommodate the ramp.

\section{RHIC Coupling Measurement and Correction}

Two projections onto two known directions are needed to determine the residual coupling. We use the same skew quadrupole families to modulation measurement and correction at RHIC. RHIC has three correction skew quadrupole families, F1,F2, F3. The contributions to the global coupling from each skew quadrupole in one family are same due to the six-fold lattice. The coupling from the three families with same strength and proper polarizations are only $120^{\circ}$ phase different. Knowing the projections onto at least two families, the residual coupling will be obtained.

The above $120^{\circ}$ difference is important to get specific residual coupling values from measurement. It holds for
RHIC at injection, store and on the ramp, if the following conditions are met:

- the betatron phase advances in every sextant are same, or almost same.

- the $Q_{x}, Q_{y}$ integer part difference equals 1 .

- the $Q_{x}, Q_{y}$ decimal part difference is small, say $\leq$ 0.015 .

\section{Orthogonal Modulations}

It is wise to choose two skew quadrupole modulation families whose coupling contributions are orthogonal. Then the correction strength is straightly obtained from the projection ratio,

$$
\left(k_{s} d l\right)_{c o r r}=-\kappa \times\left(k_{s} d l\right)_{a m p, m o d u}
$$

For RHIC the coupling contributions of three skew quadrupole are $120^{\circ}$ apart, so it is easy to construct two orthogonal families. For example, we could modulate F1 and F3 simultaneously with same frequency and phase to produce a coupling normal to that only from F2 modulation.

\section{Two Frequency Modulations}

In order to shorten the time occupied by the measurement, we could modulate two skew quadrupole families with two different modulation frequencies at the same time. From analytical solution and simulation, it shows there are more peaks in $\left(Q_{2}-Q_{1}\right)^{2}$ FFT plot. If the two modulation frequencies are $f_{1}$ and $f_{2}$, there will be peaks with following frequencies in the $\left(Q_{2}-Q_{1}\right)^{2}$ FFT plot: $f_{1}, 2 f_{1}, f_{2}$, $2 f_{2}$, and $\left|f_{2} \pm f_{1}\right|$. The modulation frequency should be chosen so that these peaks do not overlap. The projection ratio defined in Eq. 11 is still valid for each modulation frequency. $\left|f_{2} \pm f_{1}\right|$ peaks are of the same amplitude, which only decided by the two induced modulating couplings.

\section{Simulation Studies}

Simulation of coupling diagnostics and corrections are done based on the smooth accelerator model. It shows that the measurement and correction work well. In order to get better coupling correction, iteration of measurement and correction is necessary.

MADX simulations of the tune shifts with skew quadrupole modulation show that the tune change due to the residual orbit in the skew quadrupoles is small compared to that from the skew quadrupole strength modulation.

The element-by-element tracking based on the RHIC model with skew quadrupole modulation is in progress.

\section{DATA ACQUISITION AND ANALYSIS}

\section{PLL Tune measurement}

The RHIC phase lock loop ( PLL ) system has been used for fast and high resolution measurement of the eigentunes 
during the skew quadrupole modulation. Its data acquisition frequency is $177 \mathrm{~Hz}$, which is much faster than the skew quadrupole modulation frequency, ranging from $0.2-$ $1.0 \mathrm{~Hz}$. The full frequency span of the $\left(Q_{1}-Q_{2}\right)^{2}$ FFT spectrum is $88.5 \mathrm{~Hz}$. Figure 3 shows the PLL readings from three different modulations of the RHIC blue ring three skew quadrupole families. Figure 4 is the zoom-in plot of Fig. 3.

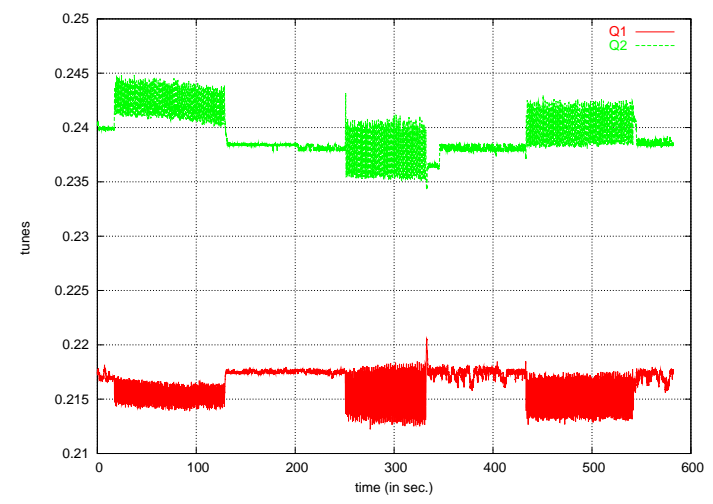

Figure 3: PLL tune readings for 3 modulations at injection.

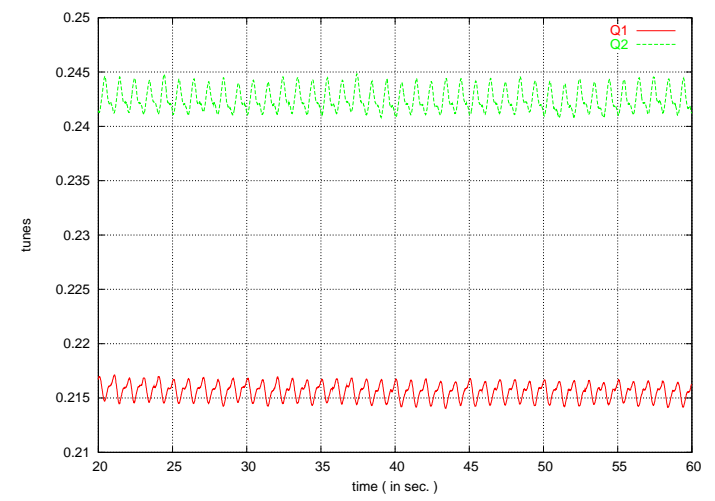

Figure 4: Zoom-in of the above PLL tune readings.

\section{FFT}

FFT is a natural choice for the data processing according to Eq. 8. However, in order to get better resolution of the FFT spectrum in the low frequency range below $2 \mathrm{~Hz}$, at least 2048 continuous sets of $Q_{1,2}$, or about 12 second of skew quadrupole modulation time is needed. In the beam experiment, 4096 continuous $Q_{1,2}$ points are normally used for FFT. Figure 5 shows the FFT of the first modulation in Fig. 3, the projection ratio from FFT is 0.622 .

\section{Linear Regression}

In order to shorten the PLL data taking time on the ramp, linear regression on Eq. 8 is used. The fitting function is assumed as:

$$
f\left(t_{i}\right)=A+B_{1} \sin \left(2 \pi f t_{i}\right)+B_{2} \cos \left(2 \pi f t_{i}\right)
$$

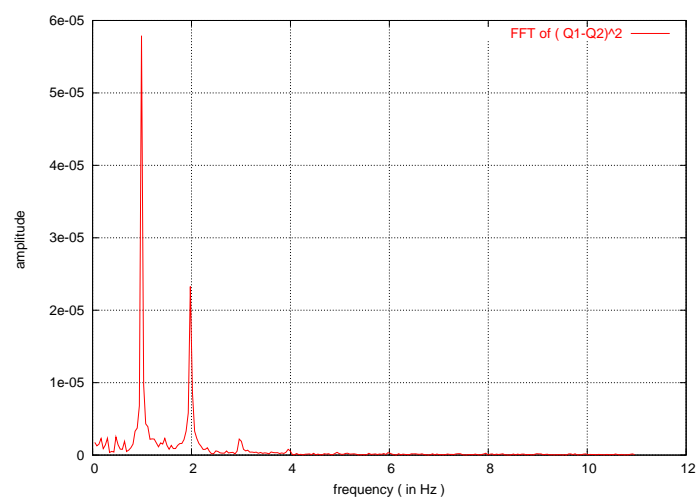

Figure 5: FFT spectrum of $\left(Q_{1}-Q_{2}\right)^{2}$ for Fig. 4 data.

$$
\begin{aligned}
& +C_{1} \sin \left(4 \pi f t_{i}\right)+C_{2} \cos \left(4 \pi f t_{i}\right) \\
& +E t_{i}+F t_{i}^{2} .
\end{aligned}
$$

We use the linear regression technique to minimize the error function $\chi^{2}$ :

$$
\chi^{2}=\sum_{i=1}^{N}\left[\left(Q_{1}-Q_{2}\right)^{2}-f\left(t_{i}\right)\right]^{2} .
$$

The projection ratio is then obtained:

$$
|\kappa|=\frac{\sqrt{B_{1}^{2}+B_{2}^{2}}}{\sqrt{C_{1}^{2}+C_{2}^{2}}} .
$$

In Eq. 13 the linear and quadratic terms of time $t_{i}$ are introduced due to a shift of the uncoupled tunes during the measurements, as observed on ramp.

Figure 6 shows the fitting result for $\left(Q_{1}-Q_{2}\right)^{2}$ from the same PLL tune measurement data as that for Fig. 5. Eight modulation PLL periods' of the raw data are used for fitting ( normally 2 or 3 modulation period's data are enough). The projection ratio obtained from fitting is 0.574. Figure 7 represents the time dependence of the projection ratios. Here the linear regression is performed every $2 \mathrm{~s}$, or 2 periods of skew quadrupole modulations. Using this method the modulation measurement time can be reduced below $10 \mathrm{~s}$ and it opens the possibility to do continuous measurement of coupling on the whole ramp. However FFT data analysis is still needed sometimes, when the PLL data qaulity is low. FFT is more robust and reveals more physics than the linear regression.

\section{MEASUREMENTS}

During the experiment time the measurement data analysis is basically off-line. Results of the measuremnts from modulations are compared with the known coupling sources, which were artificially induced into a well decoupled machine. Much more experiment time was given to measurements on ramp since there good quality PLL data were not easy to get. An on-line application is being developed that will help operational development in Run 2005. 


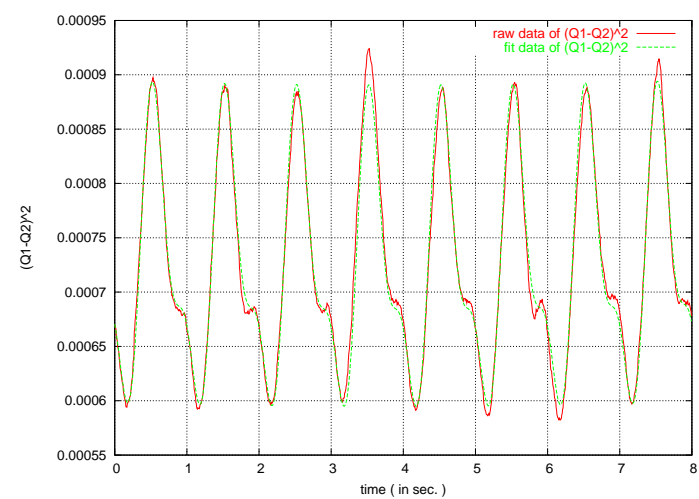

Figure 6: Linear regression of $\left(Q_{1}-Q_{2}\right)^{2}$ at injection.

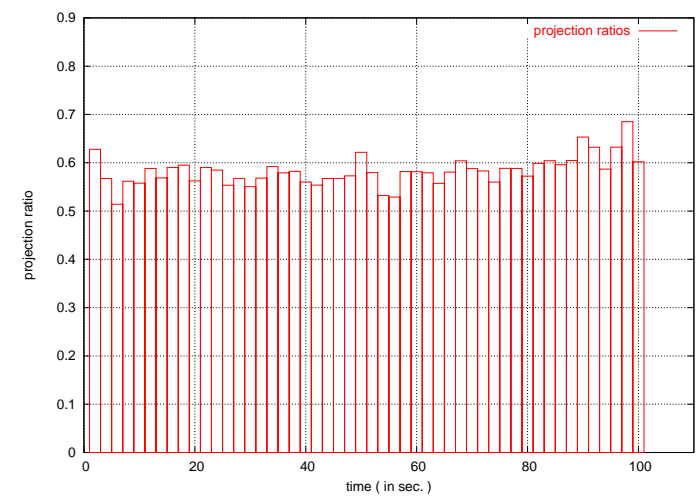

Figure 7: Continuous measurements at injection.

\section{Measurements at injection}

Every RHIC ring has 3 skew quadrupole correction families. Table 1 shows one example of coupling measurement with the three families. In the table the coupling strengths are given in current unit A. The top block of Table 1 gives residual coupling projection amplitudes and directions on the three families. The bottom block of Table 1 gives the residual couplings calculated from the two above measured projections in the three measurements. The residual couplings from different two projection combinations show good agreement in strength and direction.

Table 1: Measurement results at injection

\begin{tabular}{|l|c|c|}
\hline Family & Amplitude(A) & Direction \\
\hline F1 & 2.04 & $289.5^{\circ}$ \\
F2 & 0.92 & $49.0^{\circ}$ \\
F3 & 3.45 & $169.7^{\circ}$ \\
\hline \hline (F1,F2) & 3.00 & $336.8^{\circ}$ \\
(F2,F3) & 3.59 & $333.8^{\circ}$ \\
(F3,F1) & 3.47 & $343.6^{\circ}$ \\
\hline
\end{tabular}

This measurements were taken in the RHIC Blue ring after changing F3's integrated strength by $-0.0004 \mathrm{~m}^{-1}$. Family F3's coupling contribution direction is $169.7^{\circ}$ from model when positively powered. The average of the mea- sured residual coupling direction is about $338.07^{\circ}$, about $10^{\circ}$ different from the direction of -F3 from the model. However, the coupling amplitude measured in this example is about 3 times of the induced coupling which should be $1 \mathrm{~A}$ in the unit of current. Some dedicated beam experiment of the coupling measurements at the injection will be carried out in the next run to find out the coupling amplitude differences between the measurements and the predications.

\section{Measurements at Store}

Table 2 gives a measurement example at store, where we modulated skew quadrupole family F1 and F3 simultaneously with same strength and phase, which produced a modulation $(\mathrm{F} 1 \mathrm{~F} 3)$ with direction orthogonal to that of family F2. The top and bottom block of Table 2 give the measure med projections before and after Family F3 integrated strength change from $-0.0004 \mathrm{~m}^{-1}$ to $-0.0002 \mathrm{~m}^{-1}$.

Table 2: Measurement results at store

\begin{tabular}{|l|c|c|}
\hline Family & $\begin{array}{c}\text { Projection(A) } \\
\text { from FFT }\end{array}$ & $\begin{array}{c}\text { Projection(A) } \\
\text { from FIT }\end{array}$ \\
\hline F2 & 0.32 & 0.32 \\
F1F3 & 0.22 & 0.19 \\
\hline \hline F2 & 1.37 & 1.48 \\
F1F3 & 2.53 & 2.45 \\
\hline
\end{tabular}

The change in strength of $\mathrm{F} 3$ of $0.0002 \mathrm{~m}^{-1}$ requires a 2.2 A change of its power supply current. From the measurement the projection changes onto F2 and F1F3 coupling direction are $\sim 1.0 \mathrm{~A}, \sim 2.2 \mathrm{~A}$, respectively. The predications from model for them are $1.1 \mathrm{~A}$ and $1.9 \mathrm{~A}$, respectively. The coupling amplitude measurement error is within $15 \%$. To reduce the measurement error, more measuremnts are necessary. In this example we didn't big coupling amplitude difference between the measurement and the predication.

\section{Measurements on the Ramp}

Coupling measurement on the ramp with the skew quadrupole modulation technique posed some challenges to the RHIC PLL system during the beam experiments last run. The observables for the skew quadrupole modulation are the eigentunes, and it is important for the PLL system to give stable and reliable tunes during the modulation. However, because of energy ramp, $\beta^{\star}$ squeezing, closed orbit changes and the induced modulating coupling, sometime PLL system couldn't keep track of the two tunes during beam experiments. Figure 8 shows an example of the PLL losing lock on the ramp, where the modulation frequency is $0.2 \mathrm{~Hz}$. Figure 9 shows the projection ratios in the measurement correspnding to the valid data in Figure 8, where the 
projection ratios increase with time is due to the constant skew quadrupole modulation amplitude on energy ramp.

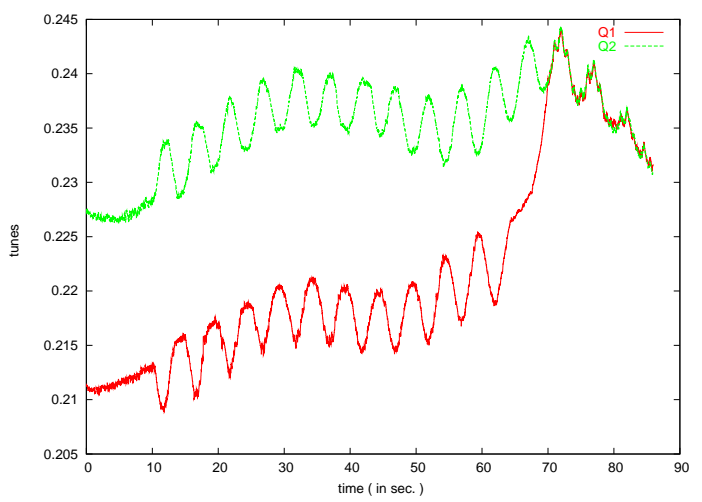

Figure 8: An example of PLL losing lock on ramp modu.

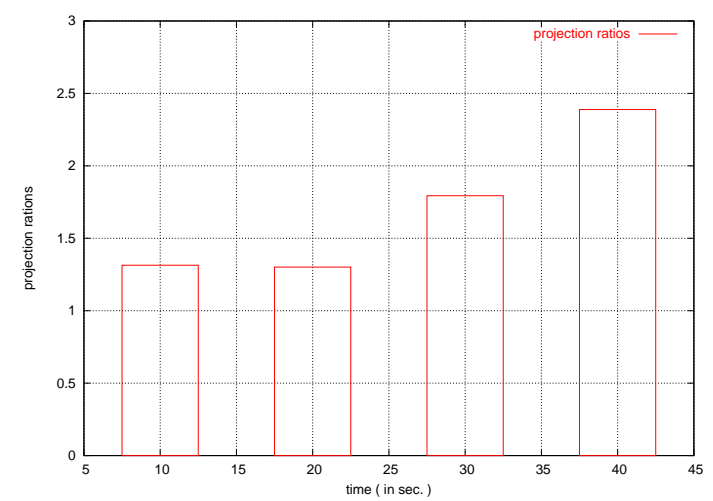

Figure 9: Projection ratios on ramp with constant skew quadrupole current modulation amplitude.

In order to reduce the demands on the RHIC PLL system, we shortened the modulation time, or lowered the skew quadrupole modulation frequencies in the beam experiments. Besides the PLL development, several attempts are under investigation. A very promising scheme coupling phase modulation, which doesn't care too much about the detailed tune data during the modulation, was put forth by Y. Luo [4] and is waiting to be tested in the next RHIC run.

\section{CONCULSION}

We introduced the principle of the skew quadrupole modulation to measure the gloable coupling. An analytical solution based on the Hamiltonian perturbation theory is obtained. Most issues about how to use this new technique for the operational measurement are also discussed. Dedicated beam experiments to test this technique were taken out in the RHIC rings in the RUN' 04. More beam experiments at the injection are needed to find out the reason for the coupling amplitude difference from the measurement and the predication. The measurement at the store shows agreements with the predication. The PLL losing lock on the ramp during the skew quadrupole moduation should be investigated. The on-line application program is in progress, which will be completed before the start of the next run. By that time, hopefully the operational global coupling measurements with the skew quadrupole modulations and the corrections based on the measurements will be carried out in the routine performance of RHIC.

\section{REFERENCES}

[1] T. Roser, private communication.

[2] G. Guignard, CERN Report No. 76-06, 1976 (unpublished).

[3] G. Guignard, Phys. Rev. E 51, p6104, 1995.

[4] Y. Luo, unpublished. 